\title{
Association behavior of thermo-responsive block copolymers based on poly(vinyl ethers)
}

\author{
Beatrice Verdonck $^{\mathrm{a}}$, Jean-François Gohy ${ }^{\mathrm{b}, 1}$, Eric Khousakoun ${ }^{\mathrm{b}}$, Robert Jérôme ${ }^{\mathrm{b}}$ and Filip Du Prez ${ }^{\mathrm{a}}$ \\ ${ }^{a}$ Department of Organic Chemistry, Polymer Chemistry Research Group, Ghent University, Krijgslaan 281 S4, \\ 9000 Ghent, Belgium \\ ${ }^{\mathrm{b}}$ Center for Education and Research on Macromolecules (CERM), University of Liège, Sart-Tilman, B6, 4000 \\ Liège, Belgium
}

\begin{abstract}
Thermo-sensitive nanosized structures have been prepared in water from poly(methyl vinyl ether)-blockpoly(isobutyl vinyl ether) (PMVE- $b$-PIBVE) block copolymers. The composition and the architecture (diblock and triblock architectures) of the PMVE- $b$-PIBVE copolymers have been varied. The investigated copolymers had an asymmetric composition with a major PMVE block. While the PIBVE blocks are hydrophobic, the PMVE blocks are hydrophilic at room temperature and become hydrophobic above their demixing temperature (around $36^{\circ} \mathrm{C}$ ) as a result of the lower critical solution temperature (LCST) behavior. At room temperature, the amphiphilic copolymers aggregate in water above a critical micelle concentration, which has been experimentally measured by hydrophobic dye solubilization. The hydrodynamic diameter of the structures formed above the cmc has been measured by dynamic light scattering (DLS) while their morphology has been studied by transmission electron microscopy (TEM). ${ }^{1} \mathrm{H}$ NMR measurements in $\mathrm{D}_{2} \mathrm{O}$ at room temperature reveal that the aggregates contain PIBVE insoluble regions surrounded by solvated PMVE chains. These investigations have shown that polydisperse spherical micelles are formed for asymmetric PMVE- $b$-PIBVE copolymers containing at least 9 IBVE units. For copolymers containing less IBVE units, loose aggregates are formed.
\end{abstract}

Finally, the thermo-responsive, reversible properties of these structures have been investigated. Above the cloud point of the copolymers, the loose aggregates precipitate while the micelles form large spherical structures.

Keywords: Block copolymers; Poly(vinyl ethers); Thermo-responsive polymers

\section{Introduction}

Stimuli-responsive block copolymers in water have been the topic of intense research during the last few years. This interest is motivated by the potential utilization of these materials for a number of technological applications, such as stimuli-controlled release of drugs [1], stimuli-controlled stabilization of emulsions [2] and [3], thermo-adjustable colloidal stabilization of organic pigments [4], etc. In water, the commonly applied stimuli are $\mathrm{pH}$, ionic strength and temperature. Block copolymers whose water-solubility of one of the constituent block can be changed by a variation of one or several of these parameters are usually considered [5]. Typical examples are copolymers containing weak polyacid or polybasic blocks that can be transformed from the hydrophilic to hydrophobic state depending on their ionization degree, block copolymer micelles that contain interpolyelectrolyte complexes as the core and that can, therefore, be disassociated by screening the electrostatic interactions, and copolymers containing blocks with a lower or upper critical solution temperature.

An illustration of $\mathrm{pH}$-responsive micellization can be found in poly(2-vinylpyridine)-block-poly(ethylene oxide) ( $\mathrm{P} 2 \mathrm{VP}-b$-PEO) copolymers that exist as unimers at $\mathrm{pH}<5$ and form micelles at higher $\mathrm{pH}$ [6-7]. Another example of $\mathrm{pH}$-driven aggregation is illustrated in double hydrophilic block copolymers based on ethylene oxide, EO, and (meth)acrylic acid, (M)AA. At low pH, hydrogen bonds exist between (M)AA and EO, that are further disrupted by an increase in $\mathrm{pH}$ resulting in the ionization of the (M)AA units [2; 8-9]. Many other related examples have recently appeared in the scientific literature.

Block copolymer micelles containing water-insoluble interpolyelectrolyte cores are a good example of ionic strength sensitive micellar systems. For example, Kataoka and co-workers investigated mixtures of fully ionized PEO- $b$-poly $(\alpha, \beta$-aspartic acid) and PEO- $b$-poly(L-lysine) diblocks [10]. Dense monodisperse micelles were formed because the oppositely charged blocks of the same length associate into interpolyelectrolyte complexes, 
that were further aggregating into micellar cores stabilized by the PEO blocks. Addition of a sufficient amount of salt resulted in the screening of the electrostatic interactions in the interpolyelectrolyte complexes and the micelles were shown to dissociate. Similar examples are found in, e.g. Ref. [11].

Thermo-sensitive block copolymer micelles have been essentially reported for systems containing a block exhibiting an LCST in water such as poly(propylene oxide) (PPO) [12], poly( $N$-isopropylacrylamide) (PNIPAM) [13] or poly( $N$-vinyl caprolactam) [14]. When this block, which is hydrophilic/hydrophobic tunable, is associated to a permanently hydrophobic block, micelles are observed below the LCST. Raising the temperature results in phase separation and reversible or irreversible micellar aggregation [15-16]. When combined with a hydrophilic block, the copolymers are soluble at a temperature below the LCST and no association is observed. At a temperature above the LCST, the thermo-sensitive blocks turn hydrophobic and micellization takes place [17-18].

Also more complex thermo-responsive copolymers consisting of two blocks presenting LCST-behavior were investigated. In addition, these systems were sensitive to $\mathrm{pH}$ and ionic strength, paving the way to multiresponsive block copolymer micelles. In this respect, Gohy et al. reported on a $\mathrm{pH}$, ionic strength and temperature sensitive system based on a mixture of P2VP- $b$-PEO and PMAA- $b$-PEO copolymers and forming different types of micellar structures depending on the applied stimulus [8]. Armes and co-workers reported on micelles based on poly(propylene oxide)-block-poly(2-(diethylamino)ethyl methacrylate) [19]. The use of such micelles allowed the switching of the inside and outside core-forming blocks by combined $\mathrm{pH}$ and temperature stimuli, leading to the so-called schizophrenic micelles. Another interesting example was reported by Laschewsky and co-workers for block copolymers combining blocks with either a LCST or a UCST. In this case, the inside and outside blocks of the micelles have been changed by a simple thermal stimulus [20].

In this contribution, we focus on block copolymers containing different poly(vinyl ether) blocks. The living cationic polymerization of vinyl ethers is now a well-established method for the design of well-defined polymer architectures [21]. In a previous paper, we have reported on the synthesis of a new class of thermo-adjustable polymer surfactants [3]. More precisely, we have combined poly(methyl vinyl ether) (PMVE) blocks with poly(isobutyl vinyl ether) (PIBVE) ones in $\mathrm{AB}, \mathrm{ABA}$ and $\mathrm{BAB}$ structures. At room temperature, the PMVE block is below its LCST. Amphiphilic materials, prone to self-association in water, are then formed. At higher temperature $\left(\sim 30-40^{\circ} \mathrm{C}\right)$, the LCST of the PMVE block is attained leading to the formation of a completely hydrophobic material.

The investigation of their surfactant properties provides crucial information with regard to the utilization of these materials in various applications, such as stabilisers, emulsifiers and dispersants. Moreover, the thermoresponsive behavior opens possibilities for designing polymer surfactants with stabilities and properties that can be tuned as a function of temperature. First, it has been demonstrated that some of the block copolymers have emulsifying properties similar or even better than those of commercial Pluronic ${ }^{\circledR}$ copolymers. Above the LCST, the emulsifying properties were considerably reduced or even disappeared. Second, the block copolymers show thermo-adjustable stabilizing activities towards hydrophobic pigment particles such as copper phthalocyanine and carbon black [4]. A detailed study of the micellization behavior of this new class of thermo-responsive surfactants as a function of the copolymer composition, polymer architecture (triblock versus diblock) and temperature is, therefore, needed, which is the purpose of the present paper. Dynamic light scattering, transmission electron microscopy, NMR and dye solubilization measurements have been applied for the characterization of a series of water soluble PMVE- $b$-PIBVE and PMVE- $b$-PIBVE- $b$-PMVE copolymers.

\section{Experimental part}

\subsection{Synthesis of the block copolymers}

The PMVE homopolymer and the PMVE-PIBVE block copolymers with well-defined structures, molecular weights and narrow polydispersities, have been synthesized by 'living' cationic polymerization techniques as described elsewhere [3] and [21]. The polymerizations were carried out with the system acetal/trimethyl silyl iodide as initiator and $\mathrm{ZnI}_{2}$ as activator. The initiating system based on diethoxyethane leads to diblock copolymers whereas the initiating system based on tetramethoxypropane leads to ABA or BAB triblock copolymers. 


\subsection{Preparation of the aqueous solutions}

$0.1 \mathrm{wt} \%(1 \mathrm{~g} / \mathrm{l})$ solution of each polymer was prepared by direct dissolution of the bulk sample into water. Bidistilled water was used and was filtered through $0.2 \mu \mathrm{m}$ syringe filters.

\subsection{Determination of cloud points}

The transmittance of the aqueous polymer solutions was monitored as a function of the temperature at $540 \mathrm{~nm}$ with a Uvikon 810 (Kontron Instruments) spectrophotometer connected to a thermostat (Julabo). The heating rate was $0.05{ }^{\circ} \mathrm{C} / \mathrm{min}$ and pure water was used as reference. Cloud point temperatures $\left(T_{\mathrm{cp}}\right)$ were defined as the temperatures at which the transmittance has dropped to $90 \%$ of the transmittance at room temperature.

\section{4. ${ }^{1}$ H NMR spectroscopy}

${ }^{1} \mathrm{H}$ NMR spectra were recorded on a Bruker AC500 FT-NMR apparatus in $\mathrm{CDCl}_{3}$ and $\mathrm{D}_{2} \mathrm{O}$ solutions at room temperature.

\subsection{Size exclusion chromatography}

SEC analyses were performed in chloroform at a flow rate of $1 \mathrm{ml} / \mathrm{min}$ with a $60 \mathrm{~cm} 10^{3} \AA$ column from Polymer Laboratories and calibrated with polystyrene standards.

\subsection{Dye solubilization measurements}

A $20 \mu \mathrm{l}$ aliquot of a $0.4 \mathrm{mM}$ solution of the dye 1,6-diphenyl-1,3,5-hexatriene (DPH) in THF was transferred to $2 \mathrm{ml}$ of an aqueous copolymer solution with different concentrations going from 0 to $2 \mathrm{~g} / \mathrm{l}$. Final concentrations of DPH and THF were $4 \mu \mathrm{M}$ and $1 \%(\mathrm{v} / \mathrm{v})$, respectively. The solutions were kept in the dark for at least 10 min before the start of the experiment. The absorption at $356 \mathrm{~nm}$ was measured as a function of the copolymer concentration, using a UV/vis spectrophotometer Uvikon 810 (Kontron Instruments). The absorption of DPH is 0.29 in a pure THF solution and 0.003 in water $(4 \mu \mathrm{M})$.

\subsection{Dynamic light scattering (DLS)}

DLS measurements were performed on a Brookhaven Instruments Corp. BI-200 apparatus equipped with a BI2030 digital correlator and an ion laser technology argon laser with a wavelength of $488 \mathrm{~nm}$. A temperaturecontrolled refractive index matching bath of filtered decaline surrounded the scattering cell. The scattering angle used for the measurements was $90^{\circ}$, and the second-order correlation function $G_{2}(t)$ was measured.

In the case of a single-exponential decay, $G_{2}(t)$ can be expressed as:

$$
G_{2}(t)=B[1+\beta \exp (-2 \Gamma t)]=B\left[1+\beta\left|G_{1}(t)\right|^{2}\right]
$$

where $B$ is the baseline, $\beta$ is an optical constant that depends on the instrument, $\Gamma$ is the decay rate for the process, $t$ is time, and $G_{1}(t)$ is the first-order correlation function, which was analyzed by the methods of the cumulants:

$$
G_{1}(t)=\exp \left[-\Gamma_{1} t+\left(\frac{\Gamma_{2}}{2 !}\right) t^{2}-\left(\frac{\Gamma_{3}}{3 !}\right) t^{3}+\cdots\right]
$$

where $\Gamma_{\mathrm{i}}$ is the $i$ th cumulant. The polydispersity index (PDI) of the aggregates was estimated from the $\Gamma_{2} / \Gamma_{1}^{2}$ ratio.

The $Z$-average diffusion coefficient over the whole set of aggregates was calculated from the first cumulant:

$$
\Gamma_{1}=D q^{2}
$$

where $D$ is the translation diffusion coefficient and $q$ is the absolute value of the scattering vector: 


$$
q=\frac{4 \pi n \sin \left(\frac{\theta}{2}\right)}{\lambda}
$$

$n$ is the refractive index of the solvent, $\theta$ is the diffusion angle, and $\lambda$ is the wavelength of the incident light.

The diffusion coefficient extrapolated to zero concentration $\left(D_{0}\right)$ for spherical particles is related to the hydrodynamic radius, $R_{\mathrm{h}}$, by the Stokes-Einstein equation:

$$
D_{0}=\frac{k_{\mathrm{B}} T}{6 \pi \eta R_{\mathrm{h}}}
$$

where $k_{\mathrm{B}}$ is the Boltzmann constant, $T$ is the absolute temperature, and $\eta$ is the viscosity of the solvent.

The mean hydrodynamic diameter $\left(D_{\mathrm{h}}=2 R_{\mathrm{h}}\right)$ and the PDI of the aggregates have been accordingly calculated. The DLS data were also analyzed by the CONTIN routine, a method which is based on a constrainted inverse Laplace transformation of the data and which gives access to a size distribution histogram for the aggregates.

\subsection{Transmission electron microscopy (TEM)}

TEM images were recorded with a Philips CM100 microscope equipped with a Gatan 673 CCD camera, and transferred to a computer equipped with the Kontron KS100 system. Samples were prepared by dipping a Formvar-coated copper grid into a dilute solution of the aggregates ( $0.1 \mathrm{wt} \%$ copolymer). The samples were stained by the vapor released by a solution of $\mathrm{RuO}_{4}$ resulting from the reaction of $\mathrm{RuCl}_{3}$ and a $5 \mathrm{wt} \%$ aqueous $\mathrm{NaClO}$ solution.

\section{Results and discussion}

In a previous paper [3], we reported on the living cationic sequential copolymerization of methyl vinyl ether (MVE) and isobutyl vinyl ether (IBVE). The copolymerization was performed by using either a mono- or bifunctional initiating system and by using either MVE or IBVE as the first monomer to be polymerized. We showed that $\mathrm{AB}, \mathrm{ABA}$ and $\mathrm{BAB}(\mathrm{A}=\mathrm{PMVE}, \mathrm{B}=\mathrm{PIBVE})$ block copolymers with narrow polydispersity indexes $\left(M_{\mathrm{w}} / M_{\mathrm{n}}<1.2\right)$ could be obtained. The total molecular weight of the prepared copolymers was kept relatively small ( $\approx 10,000$ daltons) with the purpose to use these samples as macro-surfactants. PMVE is a hydrophilic block at room temperature while it reaches its LCST and becomes hydrophobic above about $30-40{ }^{\circ} \mathrm{C}$ [22].

These key features of PMVE are also illustrated in a recent paper from Faust and co-workers [23]. These authors have synthesized linear and heteroarm star AB block copolymers containing PMVE and polyisobutylene (PIB) blocks. The total molecular weight of the copolymers as well as the composition (PMVE/PIB molar ratio) were kept constant in order to study the influence of the copolymer architecture on the micellization behavior in water.

In our previous study, it was found that the PMVE-PIBVE block copolymers were barely water-soluble with cloud points close to room temperature. These observations are consistent with the results of Faust [24] and Armes [25] for other PMVE-based block copolymers. In order to investigate the micellization behavior of these block copolymers in water, we selected for this study highly asymmetric AB and ABA block copolymers with a major PMVE block. The molecular characteristic features of the investigated copolymers, with a content of IBVE less than $10 \mathrm{~mol} \%$, are listed in Table 1.

Furthermore, we have decreased the length of the hydrophobic PIBVE block to a few units in order to determine the critical hydrophobic block length for micelle formation. In the first part of the paper, the micellization behavior of the investigated copolymers is studied while a second part is devoted to their thermo-responsive behavior. 
Table 1. Molecular characteristic features of the investigated (co)polymers

\begin{tabular}{|c|c|c|c|c|}
\hline Acronym $^{a}$ & $\begin{array}{l}\text { mol\% } \\
\text { IBVE }\end{array}$ & $\begin{array}{l}M_{\mathrm{n}} \text { PMVE block } \\
(\mathrm{g} / \mathrm{mol})\end{array}$ & $\begin{array}{l}M_{\mathrm{n}} \text { PIBVE } \text { block }^{\mathrm{b}} \\
(\mathrm{g} / \mathrm{mol})\end{array}$ & $M_{\mathrm{w}} / M_{\mathrm{n}}^{\mathrm{c}}$ \\
\hline $\mathrm{PMVE}_{85}-b-\mathrm{PIBVE}_{9}$ & 10 & 5000 & 900 & 1.15 \\
\hline $\mathrm{PMVE}_{65}-b-\mathrm{PIBVE}_{10}-b-\mathrm{PMVE}_{65}$ & 7 & 7500 & 1000 & 1.18 \\
\hline $\mathrm{PMVE}_{160}-b-\mathrm{PIBVE}_{5}$ & 3 & 9300 & 500 & 1.15 \\
\hline $\mathrm{PMVE}_{205}$ & - & 12,000 & - & 1.25 \\
\hline
\end{tabular}

${ }^{a}$ The number after the abbreviation of each polymer segment indicates the corresponding degree of polymerization.

${ }^{\mathrm{b}}$ Determined by ${ }^{1} \mathrm{H}$ NMR.

${ }^{\mathrm{c}}$ Determined by $\mathrm{SEC}$ (solvent $\mathrm{CHCl}_{3}$, polystyrene standards).

\subsection{Micellization behavior at room temperature}

Due to their highly asymmetric composition and to the hydrophobic character of the PIBVE blocks at room temperature, we expect the formation of micelles or aggregates above a critical micelle concentration $(\mathrm{cmc})$ whenever these samples are dissolved in water. In order to determine the cmc, the UV-VIS spectrum of a dye, 1,6-diphenyl-1,3,5-hexatriene or DPH, has been monitored as a function of the copolymer concentration. It has been reported earlier that the characteristic absorption band centered at $365 \mathrm{~nm}$ for DPH is sensitive to the environment and increases as the dye is localized in a hydrophobic environment [26-28].

Fig. 1 shows the evolution of the absorbance at $365 \mathrm{~nm}$ for DPH as a function of the copolymer concentration for the three investigated samples.

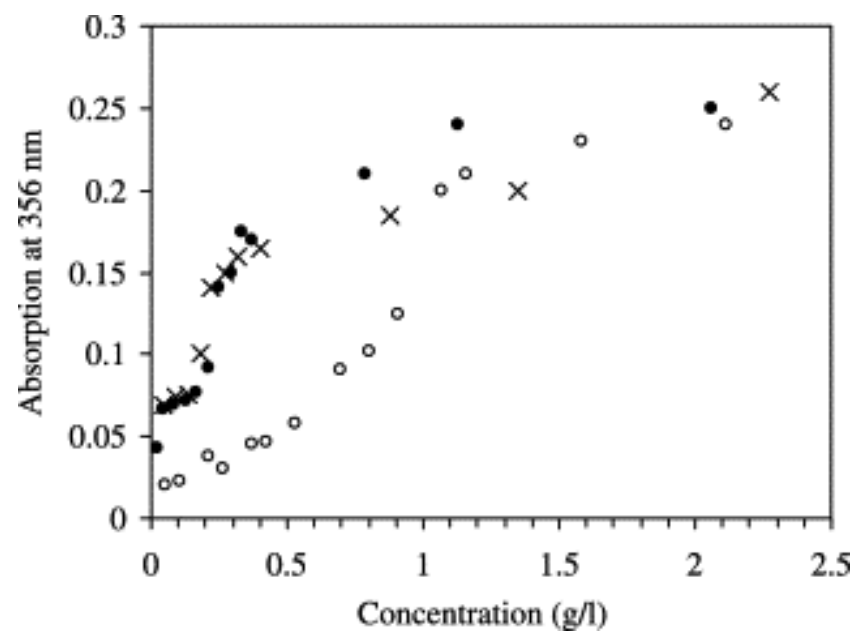

Fig. 1. Absorption at $356 \mathrm{~nm}$ of DPH aqueous solutions as a function of copolymer concentration with $P M V E_{85^{-}}$ $b-P I B V E_{9}(\bullet), P M V E_{65}-b-P I B V E_{10}-b-P M V E_{65}(\times)$ and $P M V E_{160}-b-P I B V E_{5}(\circ)$ block copolymers.

All these curves show the same characteristic behavior. The increase in absorption indicates that the solubilization of DPH is occurring into hydrophobic domains that are likely micellar cores. The cmc has been calculated from the intercept of the tangents associated to the first part (i.e. the non-associated copolymer chains) and to the second part (i.e. the steep increase in absorbance after the onset of micellization) of the sigmoidal curves (Fig. 1). A plateau is observed for the third part of the sigmoid, indicating that all the DPH molecules are located in the same hydrophobic environment.

The accordingly determined cmc values are listed in Table 2 and are very similar for the $\mathrm{PMVE}_{65}-b-\mathrm{PIBVE}_{10}-b-$ PMVE $_{65}$ and PMVE $_{85}-b-$ PIBVE $_{9}$ samples $(\sim 0.15 \mathrm{~g} / 1)$ while the cmc of the PMVE $160-b$-PIBVE 5 sample is much higher $(0.7 \mathrm{~g} / \mathrm{l})$. These values are in agreement with those of other low molecular weight amphiphilic copolymers 
with a large hydrophilic block [29]. Moreover, the cmc value is not very sensitive to the length of the PMVE block while it critically depends on the number of IBVE units. A lower number of hydrophobic units results in a higher $\mathrm{cmc}$, which is in agreement with the results reported elsewhere on Pluronic ${ }^{\circledR}$ copolymers [12].

Table 2. Characteristic features of the micelles/aggregates formed by the investigated samples at $25^{\circ} \mathrm{C}$

\begin{tabular}{|l|l|l|l|l|}
\hline Acronym & $\mathbf{c m c}(\mathbf{g} / \mathbf{l})$ & $\boldsymbol{D}_{\mathbf{h}}(\mathbf{n m})$ & PDI $^{\mathrm{a}}$ & $\boldsymbol{D}_{\text {TEM }}(\mathbf{n m})$ \\
\hline PMVE $_{85} b-\mathrm{PIBVE}_{9}$ & 0.16 & 36 & 0.22 & $32 \pm 8$ \\
\hline PMVE $_{65} b-\mathrm{PIBVE}_{10}-b-\mathrm{PMVE}_{65}$ & 0.14 & 41 & 0.24 & $40 \pm 10$ \\
\hline $\mathrm{PMVE}_{160}-b-\mathrm{PIBVE}_{5}$ & 0.7 & 80 & 0.18 & $75 \pm 5$ \\
\hline
\end{tabular}

${ }^{a}$ Polydispersity index determined by DLS.

The micelles or aggregates formed at room temperature above the cmc are assumed to consist of a PIBVE hydrophobic core surrounded by a water-soluble PMVE corona. This has been demonstrated by ${ }^{1} \mathrm{H}$ NMR spectroscopy experiments on the copolymers dissolved in deuterated chloroform $\left(\mathrm{CDCl}_{3}\right)$ and in deuterated water $\left(\mathrm{D}_{2} \mathrm{O}\right)$ (Fig. 2).

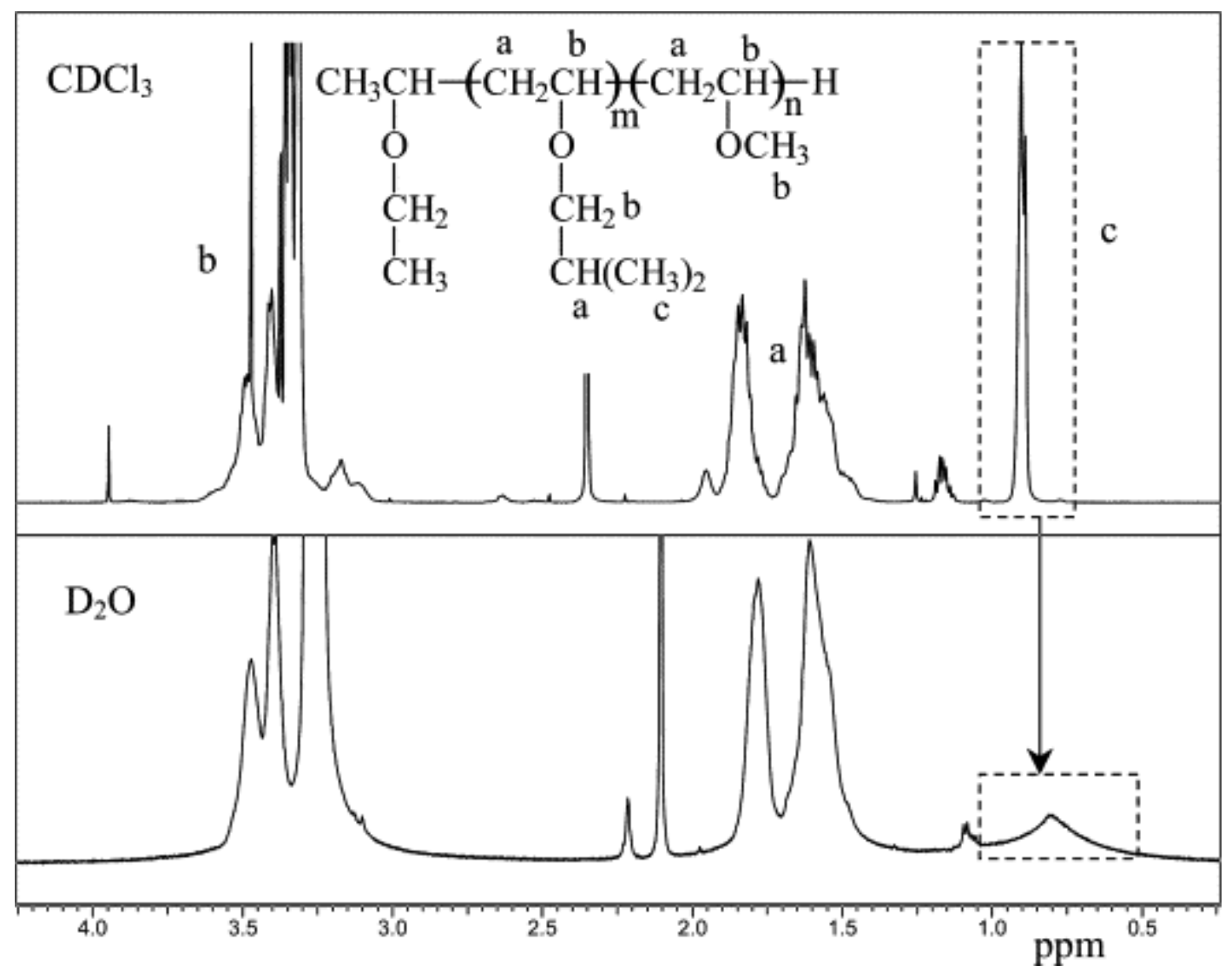

Fig. 2. ${ }^{1} \mathrm{H} N M R$ spectra of $P M V E_{85}-b-P I B V E_{9}$ in $\mathrm{CDCl}_{3}$ and $\mathrm{D}_{2} \mathrm{O}(10 \mathrm{mg} / \mathrm{ml})$.

This technique has been proved to be suitable for the detection of micellization, as exemplified by Armes and others [27] and [30]. The spectrum measured in $\mathrm{CDCl}_{3}$ shows the characteristic signals of both polymer segments. On the other hand, in $\mathrm{D}_{2} \mathrm{O}$, which is a non-solvent for PIBVE, PIBVE forms the core of the micelle and the intensity of corresponding signals vanishes in the NMR spectrum.

Dynamic light scattering (DLS) has been used to obtain information about the characteristic dimension of the structures formed by the three investigated copolymers above their cmc. Hydrodynamic diameters $\left(D_{\mathrm{h}}\right)$ 
compatible with a micellar structure have been measured for the $\mathrm{PMVE}_{85}-b-\mathrm{PIBVE}_{9}$ and $\mathrm{PMVE}_{65}-b-\mathrm{PIBVE}_{10}-b-$ PMVE $_{65}$ samples (Table 2).

The Contin size distribution histograms of these two samples are shown in Fig. 3(A) and (B) and are in agreement with the formation of micelles with a rather broad polydispersity in size (PDI indexes are listed in Table 2). In sharp contrast, much larger structures were detected for the $\mathrm{PMVE}_{160}-b-\mathrm{PIBVE}_{5}$ sample, which are indeed too large to fit to classical core-corona micelles. Moreover, the scattered light intensity is rather weak for these objects, which should, therefore, represent rather loose structures. Only one single broad population of objects is again observed in the Contin analysis as shown in Fig. 3(C).
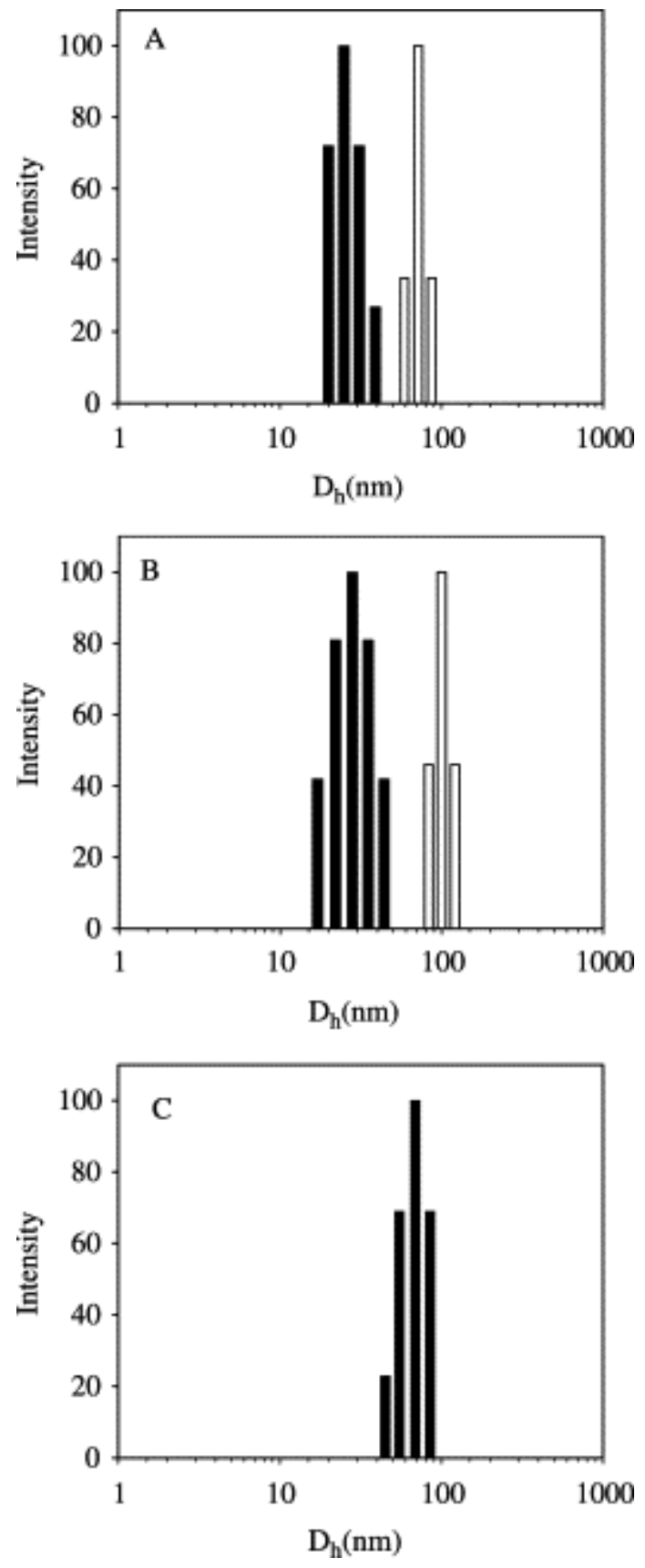

Fig. 3. CONTIN size distribution histograms observed at $25^{\circ} \mathrm{C}$ (black) and $40{ }^{\circ} \mathrm{C}$ (white) for (A) $P M V E_{85}-b$ $P I B V E_{9},(B) P M V E_{65}-b-P I B V E_{10}-b-P M V E_{65}$, (C) $P M V E_{160}-b-P I B V E_{5}$ micelles or aggregates in water $(c=1 \mathrm{~g} / l)$.

This compares with the findings of Eisenberg et al. [31], who determined a critical micellization length of 2-3 styrene units necessary for the micellization of PS- $b$-poly(sodium acrylates) block copolymers. Armes and coworkers [27] found that an amphiphilic PIBVE-b-poly(methyl tri(ethylene glycol) vinyl ether) diblock 
copolymer with only 2 IBVE units was unable to form micelles in water and existed as unimers, in contrast to the loose aggregates observed for the $\mathrm{PMVE}_{160}-b$-PIBVE 5 sample.

In a recent investigation on thermo-responsive systems formed by PS- $b$-PNIPAM diblock copolymers in water, Tenhu and co-workers reported on the formation of core-shell micellar structures for copolymers containing minor PS blocks [32]. Whenever the molar content of hydrophobic PS blocks was very small (12 mol\%), large loose structures were however, observed. They were attributed to non-structured aggregates in which the hydrophilic PNIPAM chains would be interconnected by the association of the short PS blocks, resulting in the formation of a PNIPAM microgel-type structure with PS domains as crosslinking points. A similar structure could build up in water for the $\mathrm{PMVE}_{160}-b-\mathrm{PIBVE}_{5}$ sample.

In order to get more detail about the structure of the micelles or aggregates formed by the three investigated copolymers, transmission electron microscopy (TEM) investigations have been carried out. For that purpose, a drop of a diluted micellar solution has been cast on a formvar-coated TEM grid. The dried micelles have been further stained by $\mathrm{RuO}_{4}$ vapor. Because $\mathrm{RuO}_{4}$ is not a selective contrasting agent for poly(vinyl ethers), the electronic contrast in the TEM pictures is rather weak. Typical TEM pictures are shown in Fig. 4.

A

$100 \mathrm{~nm}$
B

$100 \mathrm{~nm}$

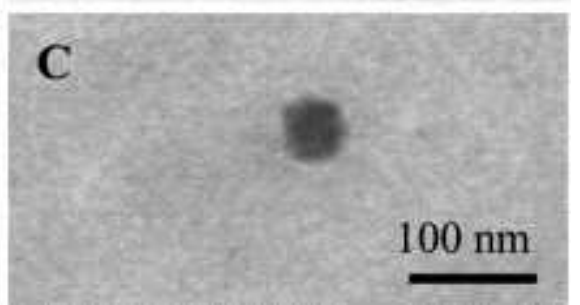

Fig. 4. TEM pictures of the dried micelles or aggregates formed by (A) $P M V E_{85}-b-P I B V E_{9}$, (B) $P M V E_{65}-b$ $P I B V E_{10}-b-P M V E_{65}$ and (C) $P M V E_{160}-b-P I B V E_{5}$ at $25^{\circ} C$.

Polydisperse spherical structures have been observed in all cases. The diameters observed for these structures are listed in Table 2 and have been estimated by observation of ca. 50 micelles collected at different places of the TEM grid. Spherical objects with a diameter typical for spherical micelles were observed for the PMVE $\mathrm{E}_{85}-b$ $\mathrm{PIBVE}_{9}$ and $\mathrm{PMVE}_{65}-b-\mathrm{PIBVE}_{10}-b-\mathrm{PMVE}_{65}$ samples. Moreover, the diameters measured from TEM pictures are in good agreement with the $D_{\mathrm{h}}$ measured by DLS (Table 2), indicating that the structure of the micelle is not much affected during the drying process on the TEM grid.

Consistently, larger spherical structures were observed for the $\mathrm{PMVE}_{160}-b$-PIBVE 5 sample with a diameter in agreement with the previous DLS measurements. The internal structure of these aggregates was observed to be ill-defined by TEM in agreement with the formation of loose aggregates (Fig. 4(C)). However, the hypothetical formation of microgel particles could not be confirmed by TEM observations on the PMVE $160-b-\mathrm{PIBVE}_{5}$ sample.

\subsection{Thermo-responsive behavior}

The thermo-responsive behavior of the micelles and aggregates prepared in water from the three investigated copolymers has been investigated. In a first step the cloud points of the aqueous solutions $(c=1 \mathrm{~g} / \mathrm{l})$ have been measured by turbidimetry. The cloud point temperature $\left(T_{\mathrm{cp}}\right)$ is defined as the temperature at which the transmittance has decreased to $90 \%$. At this temperature the solution starts to become opaque. In this way, the 
relationship between the onset of the turbidity and the corresponding increase in $D_{\mathrm{h}}$ and scattered intensity can be investigated. The measured $T_{\mathrm{cp}}$ are listed in Table 3 and are located around $30-40{ }^{\circ} \mathrm{C}$, in agreement with the LCST of the PMVE block.

Table 3. Characteristic features of the micelles/aggregates formed by the investigated samples at $40{ }^{\circ} \mathrm{C}$

\begin{tabular}{|l|l|l|l|l|}
\hline & $\boldsymbol{T}_{\mathbf{c p}}\left({ }^{\circ} \mathbf{C}\right)$ & $\boldsymbol{D}_{\mathbf{h}}(\mathbf{n m})$ & PDI & $\boldsymbol{D}_{\text {TEM }}(\mathbf{n m})$ \\
\hline $\mathrm{PMVE}_{85}-b-\mathrm{PIBVE}_{9}$ & 39 & 80 & 0.13 & $90 \pm 10$ \\
\hline $\mathrm{PMVE}_{65}-b-\mathrm{PIBVE}_{10}-b-\mathrm{PMVE}_{65}$ & 41 & 100 & 0.05 & $120 \pm 30$ \\
\hline $\mathrm{PMVE}_{160}-b-\mathrm{PIBVE}_{5}$ & 32 & - & - & $300 \pm 50$ \\
\hline PMVE $_{205}$ & 35 & - & - & - \\
\hline
\end{tabular}

The dependence of the DLS signal has been further monitored as shown in Fig. 3 and Fig. 5 . Fig. 5 shows the evolution of the mean $D_{\mathrm{h}}$ as a function of temperature for the three investigated samples. For the sake of comparison, the data obtained for a PMVE homopolymer have been plotted in the same graph.
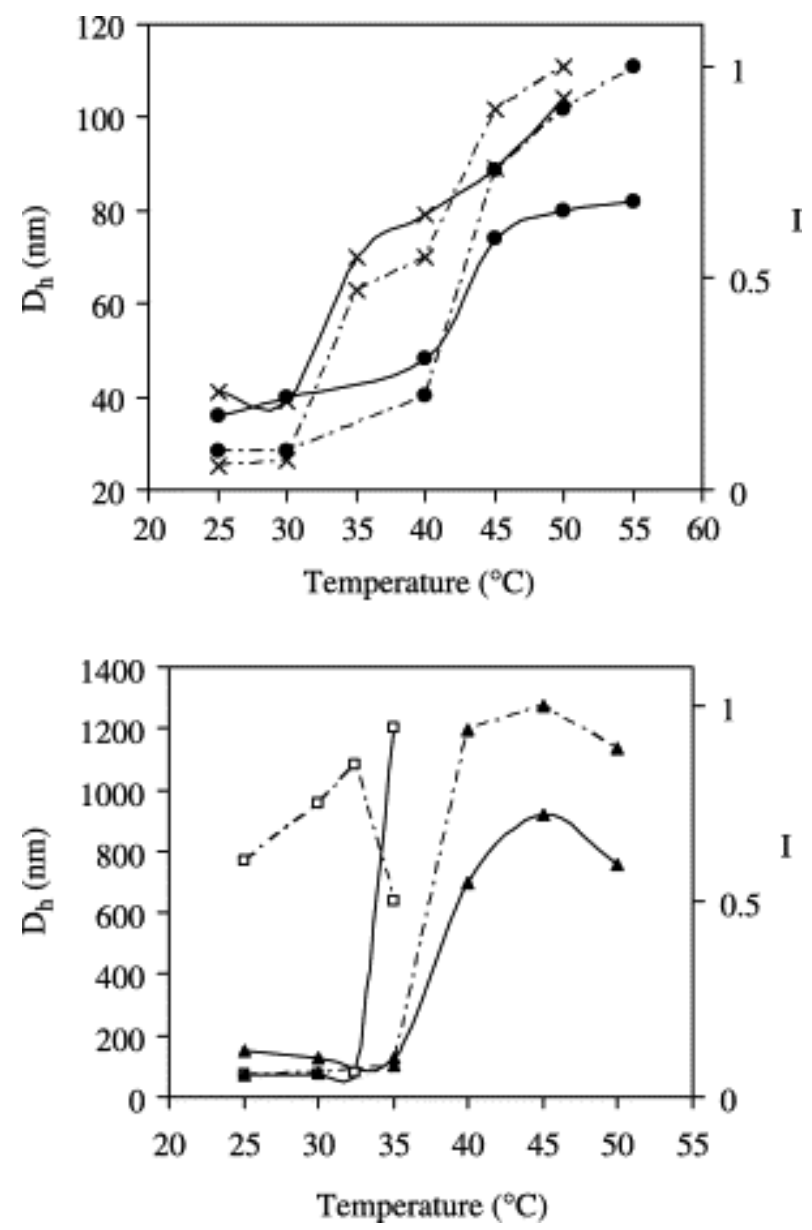

Fig. 5. Evolution of $D_{h}$ (black solid lines) and normalized scattered light intensity (I) (dotted lines) as a function of temperature for the (•) $P M V E_{85}-b-P I B V E_{9} ;(\times) P M V E_{65}-b-P I B V E_{10}-b-P M V E_{65}$; $(\square) P M V E_{160}-b-P I B V E_{5}$ micelles or aggregates and for the PMVE homopolymer $(\boldsymbol{\Lambda})$ dissolved in water $(c=1 \mathrm{~g} / \mathrm{l})$.

Both the $\mathrm{PMVE}_{85}-b$-PIBVE 9 and $\mathrm{PMVE}_{65}-b-\mathrm{PIBVE}_{10}-b$-PMVE 65 samples show a similar behavior: above a critical temperature, which is in good agreement with the macroscopic observation of the cloud point, an 
increase in $D_{\mathrm{h}}$ is noted. A plateau in $D_{\mathrm{h}}$ is then observed at higher temperature. The thermo-responsive changes were found to be entirely reversible without hysteresis.

Furthermore, the desolvation of the PVME coronal blocks above $T_{\mathrm{cp}}$ should result in a collapse of the corona and the $D_{\mathrm{h}}$ of the micelles should decrease accordingly. This is obviously not the situation observed for the PMVE $\mathrm{P}_{85^{-}}$ $b$-PIBVE 9 and $\mathrm{PMVE}_{65}-b-\mathrm{PIBVE}_{10}-b-\mathrm{PMVE}_{65}$ micelles. In the recent investigation of Tenhu et al. on PS- $b$ PNIPAM micelles [33], the desolvation of the PNIPAM coronal chains (independently evidenced by microcalorimetry measurements) was shown to have almost no effect on the $D_{\mathrm{h}}$ of the micelles. The authors suggested that the coronas of the micelles were crowded in a way that could hinder the relaxation of the PNIPAM chains. A further collapse of the micelles was shown to occur slowly at temperatures higher than the $T_{\mathrm{cp}}$ of PNIPAM. In their investigation on PMVE-containing copolymers, Faust and co-workers observed a thermo-sensitive behavior quite similar to our observations [23]. Indeed, the $D_{\mathrm{h}}$ of the micelles increased from ca. 30 to $65 \mathrm{~nm}$ at the critical temperature.

The Contin size distribution histograms associated to the aggregates, resulting from the collapse of the micelles, are shown in Fig. 3 and are in agreement with the formation of a single type of aggregate with a moderate size polydispersity. Actually, the size polydispersity (PDI) of the micelles, as measured by DLS, was systematically found to decrease above the critical temperature. The aggregated structures seem, therefore, to be more uniform in size than the starting micelles. Nevertheless this trend has not been confirmed in TEM observations (Table 3 ). This could however, suggest that the structures observed after the critical temperature are resulting from the formation of a new type of micelle rather than a simple clustering of the individual micelles. Chang et al. [34] reported large aggregates of micelles that retain a relatively narrow size distribution above the cloud point while Faust and Schuch [35] suggested the presence of regular superstructures, e.g. vesicles. It should however, be noted that the PDI of the micelles prepared by Faust and co-workers were narrower than ours.

The evolution of the scattered intensity $(I)$ as a function of temperature is plotted in Fig. 5. An important increase in $I$ is systematically observed after the critical temperature and could be attributed to either the desolvation of the PMVE blocks or the further aggregation of the micelles into larger structures.

The aggregated structures formed by the $\mathrm{PMVE}_{85}-b-\mathrm{PIBVE}_{9}$ and $\mathrm{PMVE}_{65}-b-\mathrm{PIBVE}_{10}-b-\mathrm{PMVE}_{65}$ samples above $T_{\text {cp }}$ have been imaged by TEM. For that purpose, a drop of a micellar solution at $40{ }^{\circ} \mathrm{C}$ has been cast on a formvar-coated grid whose temperature was also set to $40^{\circ} \mathrm{C}$. The preparation was carried out in an oven at $40^{\circ} \mathrm{C}$. The accordingly prepared samples have been observed by TEM as shown in Fig. 6 .

Polydisperse spherical objects are observed for both the PMVE $85-b-\mathrm{PIBVE}_{9}$ and $\mathrm{PMVE}_{65}-b-\mathrm{PIBVE}_{10}-b-\mathrm{PMVE}_{65}$ samples. Their characteristic diameters are listed in Table 3 and are roughly in agreement with DLS data. However, the TEM images were not sufficiently clear to see any internal structure. Therefore, it is not clear whether these structures result from the clustering of individual micelles or whether the individual micelles rearrange into another type of structure above the critical temperature.

The evolution of the DLS signal for the $\mathrm{PMVE}_{160}-b-\mathrm{PIBVE}_{5}$ sample is plotted in Fig. 5. A dramatic increase in turbidity can be observed as the temperature increases above $T_{\mathrm{cp}}\left(32^{\circ} \mathrm{C}\right)$. At the same critical temperature, a huge increase in $D_{\mathrm{h}}$ is observed, leading to macroscopic precipitation. This behavior is quite similar to that measured for the PMVE homopolymer (Fig. 5). The thermo-responsive properties of these structures are thus controlled by the PMVE chains and the whole system thus behaves similarly to a PMVE homopolymer. 

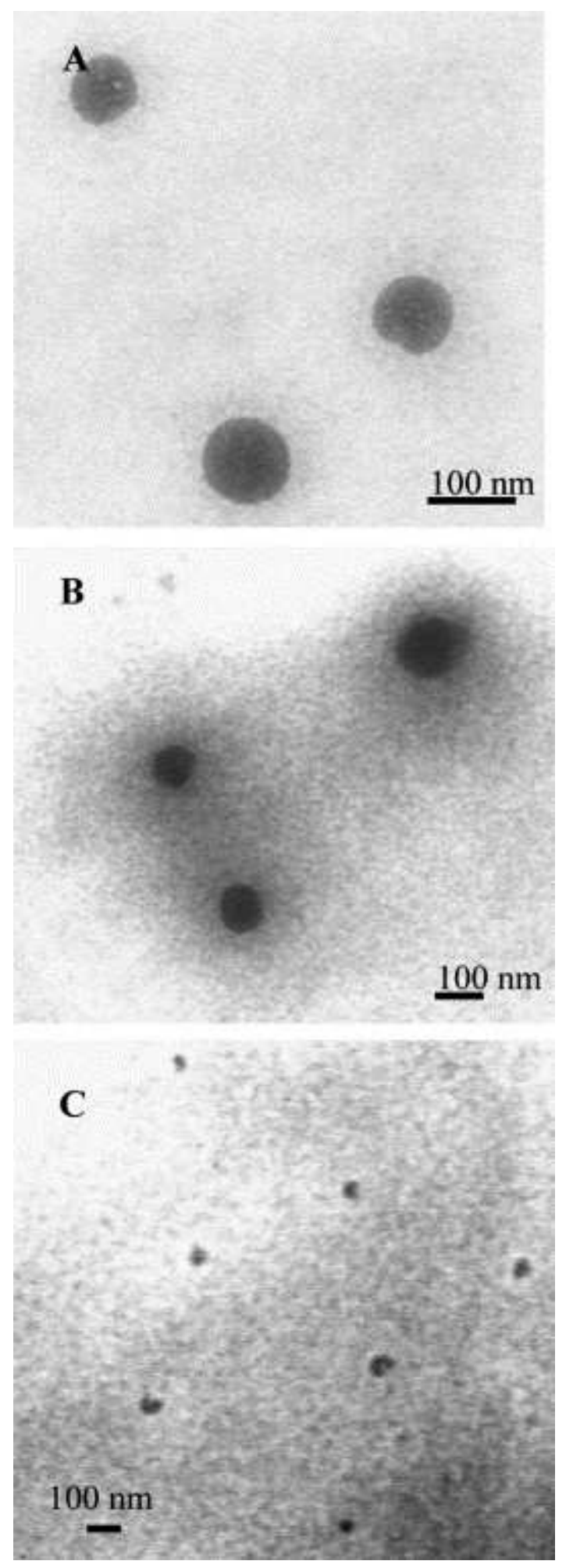

Fig. 6. TEM pictures of the dried aggregates formed by the (A) $P M V E_{85}-b-P I B V E_{9}$, (B) $P M V E_{65}-b-P I B V E_{10}-b-$ $P M V E_{65}$ and (C) $P M V E_{160}-b-P I B V E_{5}$ at $40^{\circ} \mathrm{C}$.

\section{Conclusions}

In this paper, the micellization behavior of thermo-sensitive PMVE- $b$-PIBVE amphiphilic copolymers with a relatively long PMVE block has been studied in water as a function of temperature. 
At room temperature, the micellization process has been evidenced by a dye-solubilization method. The critical micelle concentration has been accordingly determined and found to decrease with the content of hydrophobic IBVE units. Polydisperse spherical micelles have been observed by DLS and TEM for some of the investigated samples. According to ${ }^{1} \mathrm{H}$ NMR measurements, these micelles consist of a PIBVE insoluble core surrounded by PMVE coronal chains. Whenever the length of the PIBVE block is very short (mean degree of polymerization of 5), no micelles are formed but large loose aggregates are observed, that are further visualized as ill-defined spherical structures by TEM in the dried state. The architecture of the copolymer, i.e. AB versus ABA (A stands for PMVE and B for PIBVE) seems to have little effect on the micellar characteristic features within the investigated range of compositions.

Whenever temperature is raised above the cloud point of the PMVE blocks, an important increase in the size of the spherical micelles is noted, which could be attributed to either the clustering of the initial micelles or a reorganization of these micelles into another morphology, e.g. rods, vesicles, etc. TEM images revealed the formation of large spherical aggregates above $T_{\mathrm{cp}}$. However, no clear information about their internal structure could be obtained. It should be noted that the initial micelles were regenerated as temperature was lowered below $T_{\mathrm{cp}}$. The thermo-sensitive behavior of the copolymer containing a very short PIBVE block was similar to the one of PMVE homopolymer, i.e. macroscopic precipitation was observed above $T_{\mathrm{cp}}$.

\section{Acknowledgements}

B.V. thanks the Institute for the Promotion of Innovation through Science and Technology in Flanders (IWTVlaanderen) for a PhD scholarship. The ESF Programme STIPOMAT is acknowledged for financial support. CERM is very much indebted to 'Belgian Science Policy' for financial support in the frame of the 'Interuniversity Attraction Poles Programme (PAI V/03)-Supramolecular Chemistry and Supramolecular Catalysis'.

\section{References}

[1] See for example (a) Nagasaki Y, Okada T, Scholz C, Iijima M, Kato M, Kataoka K. Macromolecules 1998;31:1473-9. (b) Allen C, Maysinger D, Eisenberg A. Colloids Surf B 1999;16: 3-27. (c) Murthy KS, Ma QG, Clark CG, Remsen EE, Wooley KL. J Chem Soc, Chem Commun 2001;773-4.

[2] Mathur AM, Drescher B, Scranton AB, Klier J. Nature 1998;392: 367-70.

[3] Verdonck B, Du Prez F, Goethals EJ. Macromol Chem Phys 2003; 204:2090-8

[4] Bulychev NA, Arutunov IA, Zubov VP, Verdonck B, Goethals EJ, Du Prez FE. Macromol Chem Phys 2003;205:2457-63.

[5] Cölfen H. Macromol Rapid Commun 2001;22:219-52.

[6] Martin TJ, Prochazka K, Munk P, Webber SE. Macromolecules 1996; 29:6071-3.

[7] Gohy JF, Mores S, Varshney SK, Zhang JX, Je'rôme R. e-Polymers 2002;21.

[8] Gohy JF, Varshney SK, Je'ro^me R. Macromolecules 2001;34:3361-6.

[9] Harada A, Kataoka K. Macromolecules 1998;31:288-94.

[10] Harada A, Kataoka K. Science 1999;283:65-7.

[11] See for example Kabanov AV, Bronich TK, Kabanov VA, Yu K, Eisenberg A. Macromolecules 1996;29:6797-802.

[12] Chu B, Zhou Z. Physical chemistry of polyoxyalkylene block copolymer surfactants. In: Nace VM, editor. Nonionic surfactants.

Polyoxyalkylene block copolymers, vol. 60. New York: Marcel Dekker; 1996.

[13] Virtanen J, Tenhu H. Macromolecules 2000;33:5970-5.

[14] Verbrugghe S, Laukkanen A, Aseyev V, Tenhu H, Winnik F, Du Prez F. Polymer 2003;44:6807-14.

[15] Koňák C, Oupicky D, Chytry V, Ulbrich K, Helmstedt M. Macromolecules 2000;33:5318-20. [16] Kohori F, Sakai K, Aoyagi T, Yokoyama M, Sakurai Y, Okano T. J Controlled Release 1998;55:87-98.

[17] Okabe S, Sugihara S, Aoshima S, Shibayama M. Macromolecules 2003;36:4099-106.

[18] Topp MDC, Dijkstra PJ, Talsma H, Feijen J. Macromolecules 1997; 30:8518-20.

[19] Liu S, Billingham NC, Armes SP. Angew Chem Int Ed 2001;40(12): 2328.

[20] Arotc, aréna M, Heise B, Ishaya S, Laschewsky A. J Am Chem Soc 2002;124:3787-93.

[21] Reyntjens W, Goethals EJ. Des Monomers Polym 2001;4:195-201.

[22] Schäfer S, Moerkerke R, Berghmans H, Koningsveld R, Dusěk K, Sŏlc K. Macromolecules 1997;30:410-6.

[23] Yun J, Faust R, Szilágyi LS, Kéki S, Zsuga M. Macromolecules 2003; 36:1717-23.

[24] Hadjikyriacou S, Faust R. Macromolecules 1996;29:5261-7.

[25] Patrickios CS, Forder C, Armes SP, Billingham NC. J Polym Sci, Polym Chem 1998;36:2547-54.

[26] Alexandridis P, Holzwarth JF, Hatoon TA. Macromolecules 1994;27: 2414-25.

[27] Patrickios CS, Forder C, Armes SP, Billingham NC. J Polym Sci, Polym Chem 1996;34:1529-41.

[28] Forder C, Patrickios CS, Armes SP, Billingham NC. Macromolecules 1996;29:8160-9.

[29] Riess G. Prog Polym Sci 2003;28:1107-70.

[30] Ito K, Masuda Y. Polym J 1983;15:443-8.

[31] Astafieva I, Khougaz K, Zhong XF, Eisenberg A. Abstracts of papers of the American Chemical Society. vol. 208 1994 [211-Poly Part 2 Aug 21].

[32] Kjoniksen AL, Nyström B, Tenhu H. Colloïds Surf A: Physicochem Eng Aspects 2003;228:75-83.

[33] Nuopponen M, Ojala J, Tenhu H. Polymer 2004;45:3643-50.

[34] Chang Y, Lee SC, Kim KT, Kim C, Reeves S, Allcock HR. Macromolecules 2001;34:269-74.

[35] Schuch H, Klingler J, Rossmanith P, Frechen T, Gerst M, Feldthusen J, et al. Macromolecules 2000;33:1734-40. 